\title{
Isotropic compression of cohesive-frictional particles with rolling resistance
}

\author{
S. Luding \\ Multi Scale Mechanics, CTW, UTwente, P.O.Box 217, 7500 AE Enschede, Netherlands
}

\begin{abstract}
Cohesive-frictional and rough powders are the subject of this study. The behavior under isotropic compression is examined for different material properties involving Coulomb friction, rolling-resistance and contact-adhesion. Under isotropic compression, the density continuously increases according to Bauers exponential law, see Ref. (Bauer 1999). However, at a certain pressure/density, the behavior qualitatively changes and the system enters a second branch - again acoording to Bauers law, but with different parameters. In conclusion, the material behavior changes between two states that are both, separately, described by a simple exponential function. The phenomenology and origin of the transition between the two states is discussed.
\end{abstract}

\section{INTRODUCTION}

Cohesive-frictional and rough powders show peculiar flow behavior due to the fact that several contact forces/torques are equally important. Friction, rolling-resistance, and contact-adhesion are active at the same time and lead to macroscopic cohesion and macroscopic friction that is not proportional to the microscopic contact parameters. Besides many experiments, Molecular Dynamics (MD) or Discrete Element Models (DEM), which solve the equations of motion for all particles in a system, are used to understand these granular media. While experiments and continuum theory deal with macroscopic material parameters, for the particle simulations, the (microscopic) contact forces are the only physical laws that have to be defined beforehand (Luding 1998; Bartels et al. 2005; Dintwa et al. 2005; Luding 2006). The present simulation results are based on the contact model in the paper by Luding (Luding 2006; Luding 2008).

For powders, as an example, the particle properties and interaction laws are inserted into a discrete particle molecular dynamics and lead to the collective behavior of the dissipative, frictional, adhesive manyparticle system. From the particle simulation, one can extract, e.g., the coordination number or the pressure of the system as a function of density (Bauer 1999; Brendel et al. 2003; Morgeneyer et al. 2006; Oquendo et al. 2009), but also velocity gradient, viscosity and other macroscopic material properties.

In the following, normal interactions, like adhesion and elasto-plastic contact deformations are used as well as friction, rolling- and torsion resistance in tangential direction. Examples of an isotropic compression test are given for which the previously defined contact model parameters are varied so that the compaction process is affected. Especially of interest is the pore-number plotted against the applied pressure, which is an important ingredient for hypoplastic type constitutive models (Bauer 1999; Oquendo et al. 2009).

\section{SOFT PARTICLE SIMULATIONS}

Particle simulations are referred to as discrete element models (DEM). For details see Refs. (Cundall and Strack 1979; Bashir and Goddard 1991; Herrmann et al. 1998; Thornton 2000; Thornton and Zhang 2001; Vermeer et al. 2001; Lätzel et al. 2003; Luding 2006; Luding 2008). The elementary units of granular materials are mesoscopic grains, which deform under stress. Since the realistic modeling of the deformations of the particles is much too complicated, we relate the interaction force to the overlap $\delta$ of two particles. In tangential direction, the forces also depend on the tangential displacement since the beginning of the contact. If all forces and torques acting on a particle, either from other particles, from boundaries or from external forces, are known, the problem is reduced to the integration of Newton's equations of motion for the translational and rotational degrees of freedom.

\subsection{Normal Contact Force Laws}

Two spherical particles $i$ and $j$, with radii $a_{i}$ and $a_{j}$, respectively, interact only if they are in contact so that 
their overlap

$$
\delta=\left(a_{i}+a_{j}\right)-\left(\boldsymbol{r}_{i}-\boldsymbol{r}_{j}\right) \cdot \boldsymbol{n}
$$

is positive, $\delta>0$, with the unit vector $\boldsymbol{n}=\boldsymbol{n}_{i j}=$ $\left(\boldsymbol{r}_{i}-\boldsymbol{r}_{j}\right) /\left|\boldsymbol{r}_{i}-\boldsymbol{r}_{j}\right|$ pointing from $j$ to $i$. The force on particle $i$, from particle $j$, at contact $c$, can be decomposed into a normal and a tangential part as $\boldsymbol{f}^{c}:=\boldsymbol{f}_{i}^{c}=f^{n} \boldsymbol{n}+f^{t} \boldsymbol{t}$, where $\boldsymbol{n} \cdot \boldsymbol{t}=0$. The tangential force leads to a torque as well as rolling and torsion, as discussed below.

The simplest normal contact force model, which takes into account excluded volume and dissipation, involves a linear repulsive and a linear dissipative force

$$
f^{n}=k \delta+\gamma_{0} v_{n},
$$

with a spring stiffness $k$, a viscous damping $\gamma_{0}$, and the relative velocity in normal direction $v_{n}=-\boldsymbol{v}_{i j}$. $\boldsymbol{n}=-\left(\boldsymbol{v}_{i}-\boldsymbol{v}_{j}\right) \cdot \boldsymbol{n}=\dot{\delta}$.

This so-called linear spring dashpot (LSD) model allows to view the particle contact as a damped harmonic oscillator, for which the half-period of a vibration around an equilibrium position with a certain contact force, can be computed (Luding 1998). The typical response time on the contact level is

$$
t_{c}=\frac{\pi}{\omega}, \quad \text { with } \omega=\sqrt{\left(k / m_{12}\right)-\eta_{0}^{2}},
$$

the eigenfrequency of the contact, the rescaled damping coefficient $\eta_{0}=\gamma_{0} /\left(2 m_{i j}\right)$, and the reduced mass $m_{i j}=m_{i} m_{j} /\left(m_{i}+m_{j}\right)$. From the solution of the equation of a half period of the oscillation, one also obtains the coefficient of restitution

$$
r=v_{n}^{\prime} / v_{n}=\exp \left(-\pi \eta_{0} / \omega\right)=\exp \left(-\eta_{0} t_{c}\right),
$$

which quantifies the ratio of normal relative velocities after (primed) and before (unprimed) the collision. For a more detailed discussion of this and other, more realistic, non-linear contact models, see Ref. (Luding 1998).

The contact duration in Eq. (3) is also of practical technical importance, since the integration of the equations of motion is stable only if the integration time-step $\Delta t_{\mathrm{MD}}$ is much smaller than $t_{c}$. Note that $t_{c}$ depends on the magnitude of dissipation: In the extreme case of an overdamped spring, $t_{c}$ can become very large (which would render the contact behavior artificial (Luding et al. 1994a)). Therefore, the use of neither too weak nor too strong dissipation is recommended.

Here we apply a variant of the linear hysteretic spring model (Walton and Braun 1986; Luding 1998; Tomas 2000; Luding 2006; Luding 2008), as an alternative to the frequently applied spring-dashpot models. This model is the simplest version of some more complicated nonlinear-hysteretic force laws (Walton and Braun 1986; Zhu et al. 1991; Sadd et al. 1993; Tomas 2000), which reflect the fact that at the contact point, plastic deformations may take place and attractive (adhesive) forces exist.

The adhesive, plastic (hysteretic) force-law was introduced and described in detail in Ref. (Luding 2008), so that we do not repeat it here. Its parameters are $k_{1}, k_{2}, k_{c}$ and the range of plastic deformation relative to the particle diameter, $\phi_{f}$.

\subsection{Tangential Contact Force Laws}

For the tangential degrees of freedom, there are three different force- and torque-laws to be implemented: (i) friction, (ii) rolling resistance, and (iii) torsion resistance, as described in Ref. (Luding 2008). The unique feature of this tangential contact model is the fact that a single procedure (subroutine) can be used to compute either sliding, rolling, or torsion resistance. The subroutine needs a velocity as input and returns the respective force or quasi-force. Below, the sliding/sticking friction model will be introduced in detail, while the rolling and torsion resistance then only have to be discussed where different from the sliding model, i.e., with respect to the material parameters and the action of forces and torques.

The material parameters for friction involve a static and a dynamic friction coefficient $\mu_{s}$ and $\mu_{d}$, a tangential elasticity $k_{t}$, and a tangential viscous damping $\gamma_{t}$. For rolling and torsion resistance, the prefactors $\mu_{r}$, and $\mu_{o}$ are used, similar to the friction coefficient - and also a dynamic and a static coefficient with the same ratio as for friction is defined. Furthermore, there is a rolling- and torsion-mode elasticity $k_{r}$ and $k_{o}$, as well as the rolling- and torsion-viscousdamping $\gamma_{r}$ and $\gamma_{o}$, as specified below in table 2 .

\subsection{Background Friction}

Note that the viscous dissipation takes place in a twoparticle contact. In the bulk material, where many particles are in contact with each other, this dissipation mode is very inefficient for long-wavelength cooperative modes of motion (Luding et al. 1994b; Luding et al. 1994a). Therefore, an additional damping with the background can be introduced, so that the total force on particle $i$ is

$$
\boldsymbol{f}_{i}=\sum_{j}\left(f^{n} \boldsymbol{n}+f^{t} \boldsymbol{t}\right)-\gamma_{b} \boldsymbol{v}_{i}
$$

and the total torque

$$
\boldsymbol{q}_{i}=\sum_{j}\left(\boldsymbol{q}^{\text {friction }}+\boldsymbol{q}^{\text {rolling }}+\boldsymbol{q}^{\text {torsion }}\right)-\gamma_{b r} a_{i}^{2} \boldsymbol{\omega}_{i},
$$

with the damping artificially enhanced in the spirit of a rapid relaxation and equilibration. The sum in Eqs. 
(5) and (6) takes into account all contact partners $j$ of particle $i$, but the background dissipation can be attributed to the medium between the particles. Note that the effect of $\gamma_{b}$ and $\gamma_{b r}$ should be checked for each simulation in order to exclude artificial over-damping.

\section{COMPACTION SIMULATION RESULTS}

In this section, a "compression" test is presented, where the particles are initially positioned on a square-lattice in a cubic system with periodic boundary conditions, in order to avoid wall effects. The system is first allowed to evolve to a disordered state, by attributing random velocities to all particles. The density is then increased by slowly increasing the particle size while the system volume $V=L^{3}$, with $L=0.025 \mathrm{~m}$, is kept constant. During the simulation, the particles are growing and quantities like density (or pore-number), coordination number, energies and pressure are reported. We tested for a few cases (with low friction) that this leads to similar behavior as keeping particles at constant size and reducing the volume, however, this need more detailed study, especially for the larger values of $\mu$ and $\mu_{r}$.

\subsection{Model System}

The systems examined in the following contain $N=$ 1728 particles with equal radii $a$. In the simulations, the radii change according to the relation

$$
\frac{d a}{d t}=g_{r} a,
$$

with the relative growth rate $g_{r}=0.2$, if not explicitly specified. The growth is stopped when a target volume fraction $\nu_{\max }$, is reached, where the volume fraction is defined as $\nu=N V(a) / V$, with the particle volume $V(a)=(4 / 3) \pi a^{3}$. The particle mass $m(a)=\rho V(a)$, with the fixed material density $\rho$, changes with the radius during the growth period. The volume fraction changes with time according to the relation

$$
\frac{d \nu}{d t}=\frac{3 \nu}{a} \frac{d a}{d t}=3 \nu g_{r},
$$

which leads to the evolution of the volume fraction $\nu=\nu_{0} \exp \left(3 g_{r} t\right)$ as function of time $t$.

\subsection{Particle and Contact properties}

The particle and material parameters are summarized in table 1 and a typical set of material parameters is given in table 2 . The choice of numbers and units is such that the particles correspond to spheres with initial radius $a_{0}=5 \mu \mathrm{m}$, growing up to a maximum radius at volume fraction $\nu_{\max }=0.75$ of $a_{\max }=$ $11.7 \mu \mathrm{m}$.

The stiffness magnitude (this is not the material bulk modulus, but a contact property) used thus appears small, but for small fragile materials it is not
Table 1: The units and the microscopic particle and contact model parameters.

\begin{tabular}{ll}
\hline Property & Symbol \\
\hline \hline Time Unit & $t_{u}$ \\
Length Unit & $x_{u}$ \\
Mass Unit & $m_{u}$ \\
\hline Initial particle radius & $a_{0}$ \\
Growth rate & $g_{r}$ \\
Particle radius & $a$ \\
Material density & $\rho$ \\
\hline Elastic stiffness & $k=k_{2}$ \\
Plastic stiffness & $k_{1} / k$ \\
Adhesion "stiffness" & $k_{c} / k$ \\
Friction stiffness & $k_{t} / k$ \\
Rolling stiffness & $k_{r} / k$ \\
Torsion stiffness & $k_{o} / k$ \\
\hline Plasticity depth & $\phi_{f}$ \\
\hline Coulomb friction coefficient & $\mu=\mu_{d}=\mu_{s}$ \\
Rolling "friction" coefficient & $\mu_{r}$ \\
Torsion "friction" coefficient & $\mu_{o}$ \\
\hline Normal viscosity & $\gamma=\gamma_{n}$ \\
Friction viscosity & $\gamma_{t} / \gamma$ \\
Rolling viscosity & $\gamma_{r} / \gamma$ \\
Torsion viscosity & $\gamma_{o} / \gamma$ \\
Background viscosity & $\gamma_{b} / \gamma$ \\
Background viscous torque & $\gamma_{b r} / \gamma$ \\
\hline
\end{tabular}

unreasonable. Note that - due to the contact model the effective stiffness and cohesion depend on the volume fraction and the external pressure. The material deformation (overlap) behavior can only be realistic if the simulations are performed so slow that rate effects are small and overlaps are not becoming too large.

Using the parameter $k=k_{2}$ in Eq. (3) leads to a typical contact duration (half-period) of, initially, $t_{c} \approx$ $2.2710^{-4} t_{u}=2.2710^{-10} \mathrm{~s}$, and at maximum size, $t_{c} \approx 8.1810^{-4} t_{u}=8.1810^{-10} \mathrm{~s}$, for a normal collision with $\gamma=0$. Accordingly, an integration time-step of $t_{\mathrm{MD}}=210^{-12} \mathrm{~s}$ is used, in order to allow for a 'safe' integration of contacts. Note that not only the normal "eigenfrequency" but also the eigenfrequencies in tangential and rotation direction have to be considered as well as the viscous response times $t_{\gamma} \approx m / \gamma$. All of the eigenfrequencies should be considerably larger than $t_{\mathrm{MD}}$, whereas the viscous response times should be even larger, so that $t_{\gamma}>t_{c}>t_{\mathrm{MD}}$. The discussion of all the effects due to the interplay between the model parameters is far from the scope of this paper, however.

\subsection{Compression simulations}

When compressing the system (by growing the particles) the first quantity of interest is the density (vol- 
Table 2: The microscopic material parameters used (Values in units of time $t_{u}$, length $x_{u}$, and mass $m_{u}$ ) if not explicitly specified. The third column contains the values in SI units.

\begin{tabular}{lll}
\hline Symbol & Values & SI units \\
\hline \hline$t_{u}$ & 1 & $1 \mu \mathrm{s}$ \\
$x_{u}$ & 1 & $10 \mathrm{~mm}$ \\
$m_{u}$ & 1 & $1 \mathrm{mg}$ \\
\hline$a_{0}$ & $5.10^{-4}$ & $5.10^{-6} \mathrm{~m}$ \\
$a(t)=a_{0} e^{g_{r} t}$ & & \\
$\rho$ & 2000 & $2000 \mathrm{~kg} / \mathrm{m}^{3}$ \\
\hline$k=k_{2}$ & 100 & $10^{8} \mathrm{~kg} / \mathrm{s}^{2}$ \\
$k_{1} / k$ & 0.2 & \\
$k_{c} / k$ & 1.0 & \\
$k_{t} / k$ & 0.2 & \\
$k_{r} / k=k_{o} / k$ & 0.2 & \\
\hline$\phi_{f}$ & 0.05 & \\
\hline$\mu=\mu_{d}=\mu_{s}$ & 1 & \\
$\mu_{r}=\mu_{o}$ & 0.1 & \\
\hline$\gamma=\gamma_{n}$ & $210^{-4}$ & $210^{-4} \mathrm{~kg} / \mathrm{s}$ \\
$\gamma_{t} / \gamma$ & 0.25 & \\
$\gamma_{r} / \gamma=\gamma_{o} / \gamma$ & 0.25 & \\
$\gamma_{b} / \gamma$ & 0.10 & \\
$\gamma_{b r} / \gamma$ & 0.05 & \\
\hline
\end{tabular}

ume fraction) $\nu$ or equivalently the pore-number

$$
e=\frac{1}{\nu}-1
$$

The second quantity is the pressure that is reached during compression, plotted as a function of the density in Fig. 1 for two different combinations of friction and rolling-resistance parameters. Note that we plot the dimensionless pressure that is approximately the average overlap relative to the particle size, i.e., a dimensionless pressure of 0.1 corresponds to an average contact deformation of order of $10 \%$. Thus, at the highest pressure, due to the wide distribution of contact overlaps and forces, some particles are considerably deformed and feel accordingly extremely high forces.

During compression, the pressure remains at a very small level, until it starts to increase strongly and nonlinearly from a certain volume fraction on. There are two regimes: (i) an initial, nonlinear regime for small pressures, and (ii) an almost linear regime for large pressures. The slow simulations (red, solid lines) lead to a somewhat smaller pressure than the fast simulations (green, dashed lines), showing the dynamic effect of the rather fast compression rate with $g_{r}=0.2$. However, since the difference between fast and slow compression is only a few percent for low pressures, and much smaller for high pressures, in this study, we will present the fast compression results only.
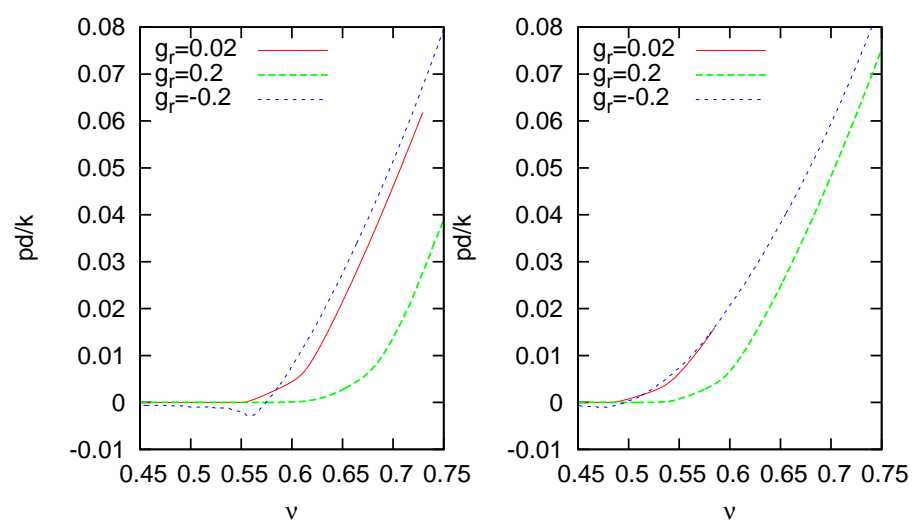

Figure 1: Dimensionless pressure $p d / k$, with $d=2 a$, plotted as function of the density for simulations with (Left) $\mu=0.01, \mu_{r}=0.1$, and (Right) $\mu=1.0, \mu_{r}=0.01$, and the other parameters as in 2 . The growth rate is given in the inset, where the negative $g_{r}=-0.2$ corresponds to unloading after the maximal density was reached.

\subsection{Parameter Study}

In the following, the friction coefficient $\mu$ and the rolling- and torsion-resistance coefficients $\mu_{r}=\mu_{o}$ are varied. The pore-number $e$ is plotted against the pressure in Fig. 2 for various simulations.

From the top panel one can conclude that small friction coefficients are always related to rather high densities, i.e., small pore numbers. Larger and larger friction coefficients, however, are not always sufficient to guarantee a lower and lower packing density, i.e., higher and higher pore number. The simulations collapse for $\mu \geq 1$.

From the bottom panel, one observes similarly that larger and larger rolling- and torsion-resistance leads to smaller densities, i.e., larger pore-numbers. On the other hand, extremely high rolling- and torsioncoefficients do not necessarily lead to lower densities. The simulations do not change anymore for $\mu_{r} \geq 0.5$.

The reason for this is a different reorganization dynamics. Increasing the friction (rolling resistance) coefficients, allows for higher pore numbers, however, above a certain value, the packing is not stabilized and finds other deformation modes to collapse. For example, when sliding is avoided (large $\mu$ ), the packing still can roll into denser positions, and similarly, when rolling is avoided (large $\mu_{r}$ ), the packing can slide into denser configurations.

\subsection{Analytical form of the porosity}

In this subsection we fit the data from the simulation with $g_{r}=0.02, \mu_{r}=0.1$, and $\mu=0.01$ using the analytical form

$$
e(p)=e_{0} \exp \left(\left[\frac{p}{h_{s}}\right]^{n_{s}}\right)
$$



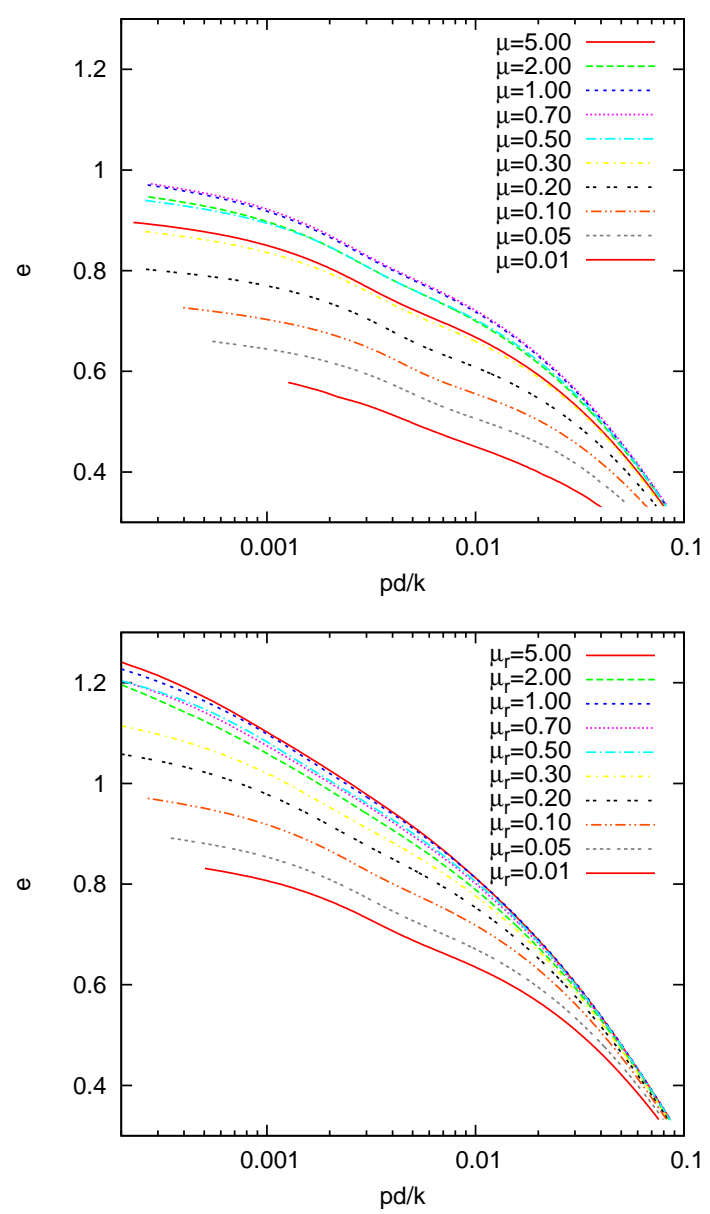

Figure 2: Pore number $e$ plotted against pressure for data with $g_{r}=0.2$ and $E_{k} / E_{p}<0.1$. The particle and contact parameters are given in table 2 , only the values of the friction coefficient are varied at constant $\mu_{r}=0.1$ (Top), and the values of rolling- and torsion-coefficients are varied at constant $\mu=1$ (Bottom).

with the hardness $h_{s}$, the maximal pore-number $e_{0}$, and a power law with exponent $n_{s}$.

Remarkably, the data are not fitted by one law only, but by two. Specifically, by fitting in the pressure ranges $p \in[20: 200], p \in[500: 2000]$, we obtain the parameters $e_{0}=0.605,0.505, h_{s}=1620,4750 \mathrm{~N} / \mathrm{m}^{2}$, and $n_{s}=0.766$, and 0.823, respectively, see Fig. 3 .

We exclude the possibility that the two regimes come from crystallization of the structure due to the monodisperse particle size distribution, by studying the pair-correlation function (Luding 2007) at different densities/pressures during compression (data not shown). The short range order (up to 4-5 particle diameters) occurs at a pressure level well below $p=100 \mathrm{~N} / \mathrm{m}^{2}$, in Fig. 3. For higher pressures, including the transition regime, there is no significant change anymore of the established structure and thus, the transition cannot be related to a transition in structure. We rather relate the transition between the two regimes to the elasto-plastic contact model, as will be discussed in more detail elsewhere. (Luding 2007).

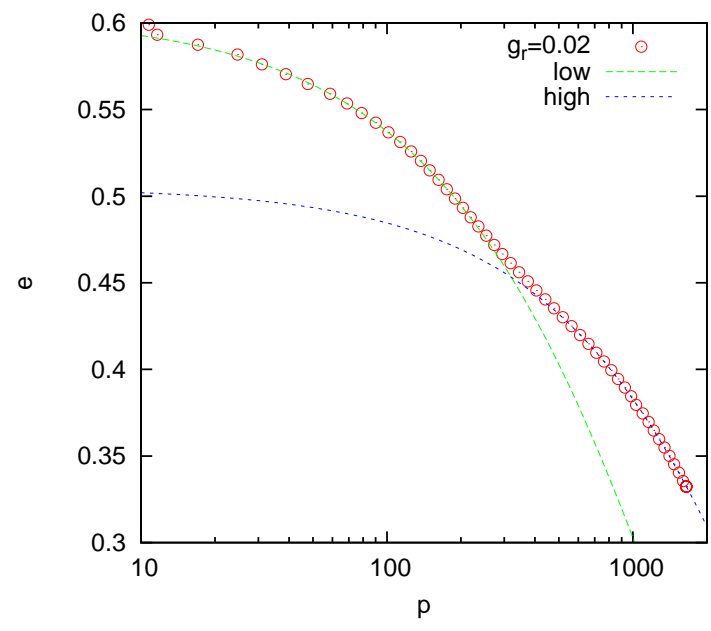

Figure 3: Pore number $e$ plotted against pressure (in units $\mathrm{N} / \mathrm{m}^{2}$ ) for data with $g_{r}=0.02, \mu=0.01$, and $\mu_{r}=0.1$. The two lines represent the fits to the low and high pressure regimes.

Given the good quality of the fit using two Bauer exponential-laws, finally, we note that the power law form proposed recently for more dynamic uni-axial compression (Brendel et al. 2003; Morgeneyer et al. 2006), does not agree that well with our data.

\section{SUMMARY AND CONCLUSION}

The present study contains compression tests of adhesive, frictional, rough powder particles. While adhesion is not varied here, both friction and rollingresistance coefficients are changed systematically. All other parameters are chosen with exemplary values, since the full set of contact models presented involves a too large number of parameters. The most relevant parameters still have to be identified and their interplay has to be better understood.

The compression behavior is well fitted by two exponential laws with different parameters, indicating two different contact mechanisms active during compression. Using friction and rolling-/torsionresistance, stable static packings could be reached with rather low densities (volume fractions) at small pressure, somewhat above $\nu_{\min } \approx 0.4$.

Eventually, the quantitative validation of the simulation contact models and the corresponding parameters the issue. The measurement of low packing fractions in adhesive, frictional fine powders is one of the possible experiments to be examined in more detail a challenge for particle contact modeling.

\section{ACKNOWLEDGEMENTS}

Valuable discussions with E. Bauer, H.-J. Butt, M. Kappl, S. McNamara, J. Tomas, and R. Tykhoniuk are acknowledged. Furthermore, we acknowledge the financial support of the Deutsche Forschungsgemeinschaft (DFG) and the Stichting voor Fundamenteel 
Onderzoek der Materie (FOM), financially supported by the Nederlandse Organisatie voor Wetenschappelijk Onderzoek (NWO).

\section{REFERENCES}

Bartels, G., T. Unger, D. Kadau, D. E. Wolf, and J. Kertesz (2005). The effect of contact torques on porosity of cohesive powders. Granular Matter 7, 139.

Bashir, Y. M. and J. D. Goddard (1991). A novel simulation method for the quasi-static mechanics of granular assemblages. J. Rheol. 35(5), 849-885.

Bauer, E. (1999). Analysis of shear band bifurcation with a hypoplastic model for a pressure and density sensitive granular material. Mechanics of Materials 31, 597.

Brendel, L., D. Kadau, D. E. Wolf, M. Morgeneyer, and J. Schwedes (2003). Compaction of cohesive powders: A novel description. AIDIC Conference Series 6, 55-65.

Cundall, P. A. and O. D. L. Strack (1979). A discrete numerical model for granular assemblies. Géotechnique 29(1), 47-65.

Dintwa, E., M. van Zeebroeck, E. Tijskens, and H. Ramon (2005). Torsion of viscoelastic spheres in contact. Granular Matter 7, 169.

Herrmann, H. J., J.-P. Hovi, and S. Luding (Eds.) (1998). Physics of dry granular media - NATO ASI Series E 350, Dordrecht. Kluwer Academic Publishers.

Lätzel, M., S. Luding, H. J. Herrmann, D. W. Howell, and R. P. Behringer (2003). Comparing simulation and experiment of a $2 \mathrm{~d}$ granular couette shear device. Eur. Phys. J. E 11(4), 325-333.

Luding, S. (1998). Collisions \& contacts between two particles. In H. J. Herrmann, J.-P. Hovi, and S. Luding (Eds.), Physics of dry granular media - NATO ASI Series E350, Dordrecht, pp. 285. Kluwer Academic Publishers.

Luding, S. (2006). About contact force-laws for cohesive frictional materials in $2 \mathrm{~d}$ and $3 \mathrm{~d}$. In P. Walzel, S. Linz, C. Krülle, and R. Grochowski (Eds.), Behavior of Granular Media, pp. 137-147. Shaker Verlag. Band 9, Schriftenreihe Mechanische Verfahrenstechnik, ISBN 38322-5524-9.

Luding, S. (2007). Contact models for very loose granular materials. In P. Eberhard (Ed.), IUTAM bookseries: Symposium on Multiscale Problems in Multibody System Contacts, Berlin, pp. 135-150. Springer.
Luding, S. (2008). Cohesive frictional powders: Contact models for tension. Granular Matter 10, 235-246.

Luding, S., E. Clément, A. Blumen, J. Rajchenbach, and J. Duran (1994a). Anomalous energy dissipation in molecular dynamics simulations of grains: The "detachment effect". Phys. Rev. E 50, 4113.

Luding, S., E. Clément, A. Blumen, J. Rajchenbach, and J. Duran (1994b). The onset of convection in molecular dynamics simulations of grains. Phys. Rev. E 50, R1762.

Morgeneyer, M., M. Röck, J. Schwedes, L. Brendel, K. Johnson, D. Kadau, D. E. Wolf, and L.-O. Heim (2006). Compaction and mechanical properties of cohesive granular media. In P. Walzel, S. Linz, C. Krülle, and R. Grochowski (Eds.), Behavior of Granular Media, pp. 107-136. Shaker Verlag. Band 9, Schriftenreihe Mechanische Verfahrenstechnik, ISBN 38322-5524-9.

Oquendo, W. F., J. D. Munoz, and A. Lizcano (2009). Oedometric test, bauers law and the micro-macro connection for a dry sand. Computer Physics Communication 180, 616-620.

Sadd, M. H., Q. M. Tai, and A. Shukla (1993). Contact law effects on wave propagation in particulate materials using distinct element modeling. Int. J. Non-Linear Mechanics 28(2), 251.

Thornton, C. (2000). Numerical simulations of deviatoric shear deformation of granular media. Géotechnique 50(1), 43-53.

Thornton, C. and L. Zhang (2001). A dem comparison of different shear testing devices. In Y. Kishino (Ed.), Powders \& Grains 2001, Rotterdam, pp. 183-190. Balkema.

Tomas, J. (2000). Particle adhesion fundamentals and bulk powder consolidation. KONA 18, 157-169.

Vermeer, P. A., S. Diebels, W. Ehlers, H. J. Herrmann, S. Luding, and E. Ramm (Eds.) (2001). Continuous and Discontinuous Modelling of Cohesive Frictional Materials, Berlin. Springer. Lecture Notes in Physics 568.

Walton, O. R. and R. L. Braun (1986). Viscosity, granular-temperature, and stress calculations for shearing assemblies of inelastic, frictional disks. J. Rheol. 30(5), 949-980.

Zhu, C. Y., A. Shukla, and M. H. Sadd (1991). Prediction of dynamic contact loads in granular assemblies. J. of Applied Mechanics 58, 341. 